\title{
Comparative effects of dried plum and dried apple on bone in postmenopausal women
}

\author{
Shirin Hooshmand ${ }^{1}$, Sheau C. Chai ${ }^{1}$, Raz L. Saadat ${ }^{1}$, Mark E. Payton ${ }^{2}$, Kenneth Brummel-Smith ${ }^{3}$ \\ and Bahram H. Arjmandi ${ }^{1 *}$ \\ ${ }^{1}$ Department of Nutrition, Food and Exercise Sciences, Florida State University, 436 Sandels Building, Tallahassee, \\ FL 32306, USA \\ ${ }^{2}$ Department of Statistics, Oklahoma State University, Stillwater, OK 74078, USA \\ ${ }^{3}$ College of Medicine, Florida State University, Tallahassee, FL 32306, USA
}

(Received 15 September 2010 - Revised 6 January 2011 - Accepted 21 January 2011 - First published online 31 May 2011)

\section{Abstract}

Aside from existing drug therapies, certain lifestyle and nutritional factors are known to reduce the risk of osteoporosis. Among the nutritional factors, dried plum or prunes (Prunus domestica L.) is the most effective fruit in both preventing and reversing bone loss. The objective of the present study was to examine the extent to which dried plum reverses bone loss in osteopenic postmenopausal women. We recruited 236 women, 1-10 years postmenopausal, not on hormone replacement therapy or any other prescribed medication known to influence bone metabolism. Qualified participants ( $n$ 160) were randomly assigned to one of the two treatment groups: dried plum $(100 \mathrm{~g} / \mathrm{d})$ or dried apple (comparative control). Participants received $500 \mathrm{mg}$ Ca plus $400 \mathrm{IU}$ (10 $\mu \mathrm{g})$ vitamin D daily. Bone mineral density (BMD) of lumbar spine, forearm, hip and whole body was assessed at baseline and at the end of the study using dual-energy X-ray absorptiometry. Blood samples were collected at baseline, 3, 6 and 12 months to assess bone biomarkers. Physical activity recall and 1-week FFQ were obtained at baseline, 3, 6 and 12 months to examine physical activity and dietary confounders as potential covariates. Dried plum significantly increased BMD of ulna and spine in comparison with dried apple. In comparison with corresponding baseline values, only dried plum significantly decreased serum levels of bone turnover markers including bone-specific alkaline phosphatase and tartrate-resistant acid phosphatase-5b. The findings of the present study confirmed the ability of dried plum in improving BMD in postmenopausal women in part due to suppressing the rate of bone turnover.

Key words: Osteoporosis: Dried plums: Clinical trials

Osteoporosis is a major public health problem in postmenopausal women. In the USA alone, eight million women have osteoporosis that results in more than one million fractures per year in women who are 45 years and older ${ }^{(1)}$. The cost of treating osteoporosis and its fractures has been estimated to be over $\$ 19$ billion/year. Although there are a number of agents available for the treatment and/or prevention of osteoporosis, some patients have contraindications to using them or prefer alternative therapies including dietary supplements and functional foods ${ }^{(2)}$. Studies have consistently shown that a higher fruit and vegetable intake has positive effects on bone mineral density (BMD) ${ }^{(3-7)}$. Muhlbauer et al. ${ }^{(8)}$ examined the effects of a number of fruits and vegetables on bone resorption by assessing the urinary excretion of ${ }^{3} \mathrm{H}$ released from bone, and showed that dried plum ${ }^{(8)}$ among fruits and onion among vegetables were the most effective functional foods with bone-modulating effects. To our knowledge, Muhlbauer et $a l .{ }^{(8)}$ were the first group who showed that prunes (dried plums) have the ability to prevent osteoporosis by inhibiting bone resorption. To follow up on Muhlbauer's findings, our laboratory conducted several animal studies and a 3-month clinical trial. The results of these studies showed that dried plum has positive effects on bone indices. The results from animal studies indicated that dried plum not only prevents ${ }^{(9)}$ but more importantly reverses bone loss in two separate models of low bone mass ${ }^{(10-12)}$. Our initial animal study ${ }^{(13)}$ indicated that dried plum prevented the ovariectomy-induced BMD reduction in the femur and lumbar vertebra. In another study, rats were ovariectomised and allowed to lose bone before the initiation of treatment to

Abbreviations: BALP, bone-specific alkaline phosphatase; BMD, bone mineral density; CRP, C-reactive protein; OC, osteocalcin; TRAP5b, tartrate-resistant acid phosphatase-5b.

*Corresponding author: Dr B. H. Arjmandi, fax +1 850645 5000, email barjmandi@fsu.edu 
mimic established osteoporosis. Dried plum intake as low as $5 \%(\mathrm{w} / \mathrm{w})$ restored BMD to the level of intact rats ${ }^{(10)}$. More importantly, dried plum reversed the loss of trabecular architectural properties such as trabecular number, connectivity density and trabecular separation, which to our knowledge is unique to dried plums in comparison with soya or its isoflavones ${ }^{(14)}$, flaxseed ${ }^{(15,16)}$, apples ${ }^{(17)}$, blueberries and strawberries (BH Arjmandi, unpublished results). According to Lane et al. ${ }^{(18)}$, once trabecular bone is lost, it would be difficult to restore it. The efficacy of dried plum in the reversal of bone loss in rat models of established osteoporosis ${ }^{(9,11,12)}$ exceeds many of the agents with bone-forming ability such as growth hormone and insulin growth factor ${ }^{(19)}$. In addition to the animal studies, the findings of our short-term clinical trial indicated that the consumption of dried plum $(100 \mathrm{~g} / \mathrm{d})$ by postmenopausal women significantly improved markers of bone turnover ${ }^{(17)}$. Our observations of serum bone markers after 3 months of dried plum consumption support the notion that dried plum significantly improves bone mass in a longer treatment period. Although the effects of dried plum on bone biomarkers are promising, a longer-term study with BMD assessments is needed to confirm the bone-protective effects of dried plum in postmenopausal women. Hence, we conducted a 1-year comparative control randomised study to examine the effects of daily consumption of $100 \mathrm{~g}$ dried plum on BMD in osteopenic postmenopausal women who were not on hormone replacement therapy or any other agents known to significantly influence bone metabolism.

\section{Experimental methods}

\section{Subjects}

A total of 236 women, 1-10 years postmenopausal, not on hormone replacement therapy for at least 3 months before the initiation of the study were recruited. Women whose BMD $t$-score at any site fell below $2.5 \mathrm{sD}$ of the mean were excluded from the study. Furthermore, subjects treated with calcitonin, bisphosphonates, raloxifene and/or anabolic agents such as parathyroid hormone and growth hormone, or steroids within 3 months before the start of the study were excluded. In addition, subjects with metabolic bone disease, renal disease, cancer, CVD, diabetes mellitus, respiratory disease, gastrointestinal disease, liver disease or other chronic diseases, heavy smoking (more than twenty cigarettes/d) and current use of any prescription medications known to alter bone and Ca metabolism were excluded. Women who regularly consumed dried plum or prune juice were not included in the study. The study was conducted according to the guidelines laid down in the Declaration of Helsinki, and all procedures involving human subjects were approved by the Institutional Review Board at the Florida State University (Tallahassee, FL, USA). Written informed consent was obtained from all subjects. A complete medical and nutritional history was obtained from the subjects before initiating the treatments. The subjects were advised to maintain their usual physical activity and diet pattern.

\section{Study design}

Of the 236 screened volunteers, 160 eligible postmenopausal women were randomly assigned to one of the two dietary treatment groups: dried plum $(100 \mathrm{~g})$ or dried apple $(75 \mathrm{~g})$. The amount of dried apple was chosen based on comparable amount of energy, carbohydrates, fat and fibre, which would be obtained from $100 \mathrm{~g}$ dried plum (Table 1$)^{(17)}$. The amount of dried plum was based on the findings of our short-term clinical trial ${ }^{(17)}$, which indicated that the consumption of $100 \mathrm{~g}$ dried plum per $\mathrm{d}$ for $90 \mathrm{~d}$ significantly modulated bone markers in postmenopausal women. Because of the known laxative properties of dried plum, the study participants were asked to gradually incorporate dried plum into their diets. The subjects were asked to return any unused dried fruit as part of monitoring compliance. All participants received $500 \mathrm{mg}$ Ca plus $400 \mathrm{IU}(10 \mu \mathrm{g})$ vitamin D daily.

Table 1. Nutrient concentration of dried apple and dried plum obtained by both calculation and actual analyses

\begin{tabular}{|c|c|c|c|c|}
\hline & \multicolumn{2}{|c|}{ Dried apple (per $75 \mathrm{~g}$ ) } & \multicolumn{2}{|c|}{ Dried plum (per $100 \mathrm{~g}$ ) } \\
\hline & Calculated $^{\star}$ & Actual† & Calculated $^{*}$ & Actual $\dagger$ \\
\hline Energy (kJ) & $1004 \cdot 83$ & $916 \cdot 90$ & $1000 \cdot 64$ & 921.09 \\
\hline Fat $(\mathrm{g})$ & 0.43 & 0.37 & 0.52 & 0.23 \\
\hline Total carbohydrates $(\mathrm{g})$ & 58.5 & 70.5 & $62 \cdot 7$ & 63.4 \\
\hline Fibre $(\mathrm{g})$ & 6 & NA & $7 \cdot 10$ & NA \\
\hline Protein $(\mathrm{g})$ & 1.5 & 0.83 & $2 \cdot 61$ & $2 \cdot 24$ \\
\hline $\mathrm{Ca}(\mathrm{mg})$ & $11 \cdot 25$ & $15 \cdot 0$ & 51.0 & 30.0 \\
\hline$P(\mathrm{mg})$ & $14 \cdot 2$ & $30 \cdot 6$ & 79 & $50 \cdot 0$ \\
\hline $\mathrm{Fe}(\mathrm{mg})$ & 1.05 & - & - & - \\
\hline Vitamin A $(\mu \mathrm{g})$ & 9 & - & - & - \\
\hline Vitamin C (mg) & 2.25 & - & - & - \\
\hline
\end{tabular}

NA, not assessed.

${ }^{*}$ Results obtained from Food Processor version 7.50 (ESHA Research, Salem, OR, USA).

† Gross energy analysed by bomb calorimetry (Parr 1261 Calorimeter; Parr Instrument Company, Moline, IL, USA), crude protein by the Association of Official Analytical Chemists Kjeldahl method, fat by diethyl ether extraction, and $\mathrm{Ca}$ and $\mathrm{P}$ content by atomic absorption spectrophotometry (Perkin-Elmer Atomic Absorption Spectrophotometer, model 5100PC; Perkin-Elmer, Norwalk, CT, USA). 
Dietary and physical activity assessment and anthropometric measurements

Medical and nutritional histories were obtained at the beginning of the study. The subjects completed a $7 \mathrm{~d}$ FFQ via interview at baseline, 3, 6 and 12 months. Nutrient analysis was performed using food analysis software (Food Processor version 7.50; ESHA Research, Salem, OR, USA). Similarly, physical activity patterns were assessed at baseline, 3, 6 and 12 months. The Five-City Project Physical Activity Recall was used to assess current physical activity, sleep and activity patterns ${ }^{(20)}$. Anthropometric data were also collected at baseline, 3, 6 and 12 months, and height and weight were used to calculate BMI $\left(\mathrm{kg} / \mathrm{m}^{2}\right)$.

\section{Bone density assessments}

Bone density was assessed at the beginning and at the end of the treatment by dual-energy X-ray absorptiometry (GE Healthcare Lunar, Madison, WI, USA) equipped with appropriate software for whole-body, lumbar spine, hip and forearm BMD. Densitometer stability was evaluated by performance of phantom scans on the dates of all data acquisition.

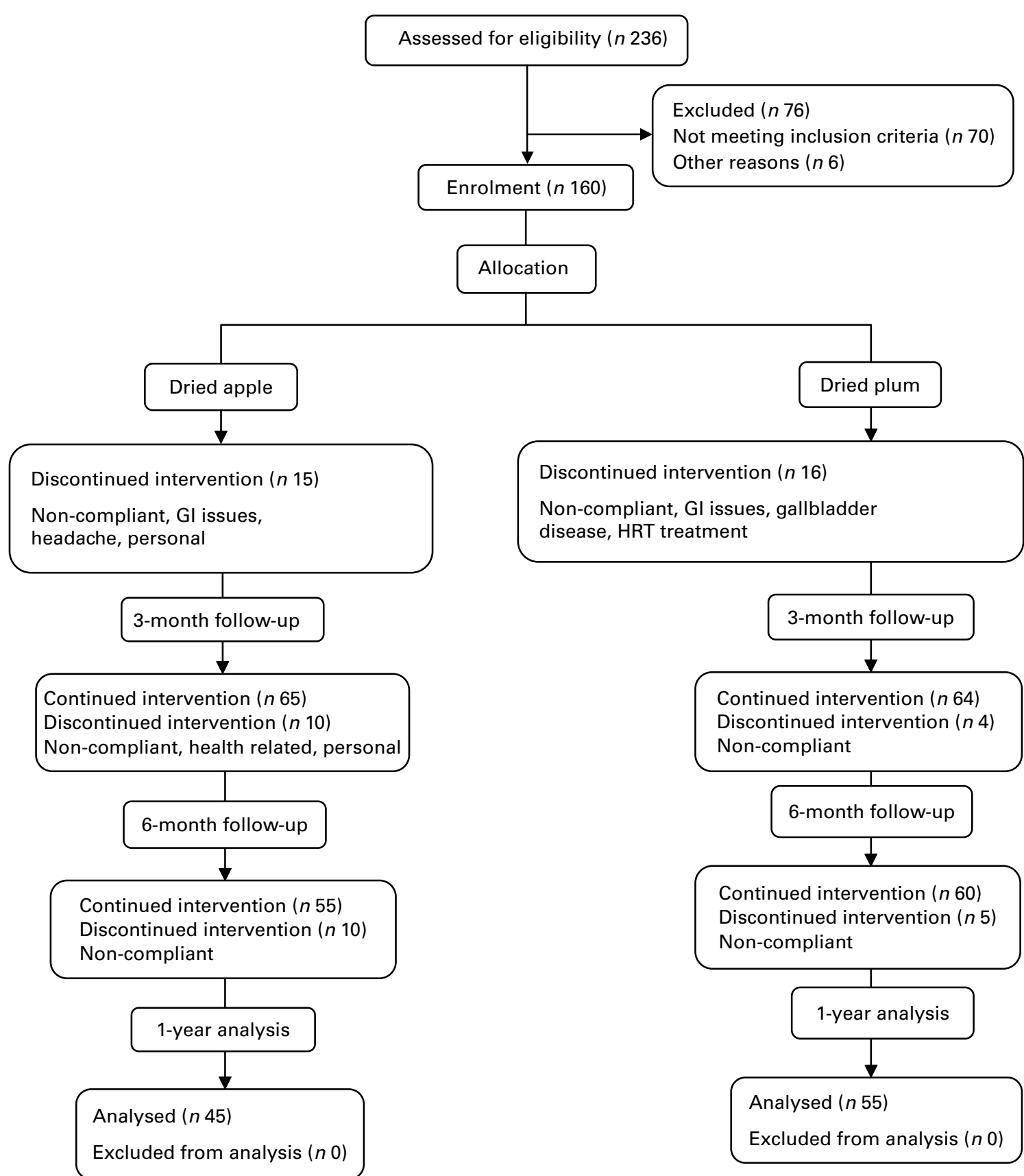

Fig. 1. Flow chart of the study design and subject participation. GI, gastrointestinal; HRT, hormone replacement therapy. 


\section{Bone marker measurements}

Venous blood samples were obtained after an overnight fast from each subject at baseline, 3, 6 and 12 months of the study for various analyses. Blood samples were centrifuged at $3500 \mathrm{~g}$ for $15 \mathrm{~min}$ at $4^{\circ} \mathrm{C}$, and serum samples were separated, divided into aliquots and stored at $-80^{\circ} \mathrm{C}$ until analyses. Bone-specific alkaline phosphatase (BALP) and osteocalcin (OC), markers of bone turnover, were measured using commercially available ELISA kits (Quidel Biosystems, Mountain View, CA, USA). Tartrate-resistant acid phosphatase-5b (TRAP5b), a marker of bone resorption in blood, was measured using ELISA kits (Quidel Biosystems). In order to determine the potential anti-inflammatory role of dried plum in modulating bone metabolism, serum C-reactive protein (CRP) was assessed using the SIRRUS clinical chemistry analyser (Stanbio Laboratory, Boerne, TX, USA) at baseline, 3, 6 and 12 months.

\section{Statistical analyses}

Data were analysed using ANOVA methods with PROC MIXED in PC SAS (version 9.1; SAS Institute, Cary, NC, USA), analysing the main and interaction effects of the two factors: treatment (dried apple or dried plum) and time (baseline or after treatment). Since each subject was measured at baseline and at various time intervals, repeated-measures model was utilised. The mean changes in each time point for the dried plum and dried apple treatment groups were compared by analysing the interaction effects of the two factors, treatment and time, using the SLICE option in an LSMEANS (leastsquares means) statement. Data are reported as least-squares means with their standard errors; unless otherwise indicated, $P<0.05$ was regarded as statistically significant.

\section{Results}

Baseline characteristics, anthropometric measurements, dietary intake and physical activity assessments

Of the 236 women, 100 (fifty-five on dried plum and forty-five on dried apple) completed the study. The attrition rates were not significantly different between the two treatment groups (37.5\%; Fig. 1). Baseline characteristic data were not significantly different for women who completed the study (Table 2).

Age, years since menopause, body weight and BMI were similar at baseline between the treatment groups. The 100 participants who remained in the study adhered to the regimens, as indicated by a self-monitoring checklist provided to them on a monthly basis and by assessing a $7 \mathrm{~d}$ FFQ. Overall, the dried fruit regimens were well accepted and considered to be palatable, as stated by the subjects. The compliance to the dried fruit regimens was on average $82 \%$ in each group. Analysis of the $7 \mathrm{~d}$ FFQ indicated that the participant's food intakes were not significantly different from their corresponding baseline values between the two treatment groups throughout the study period (Table 3 ).

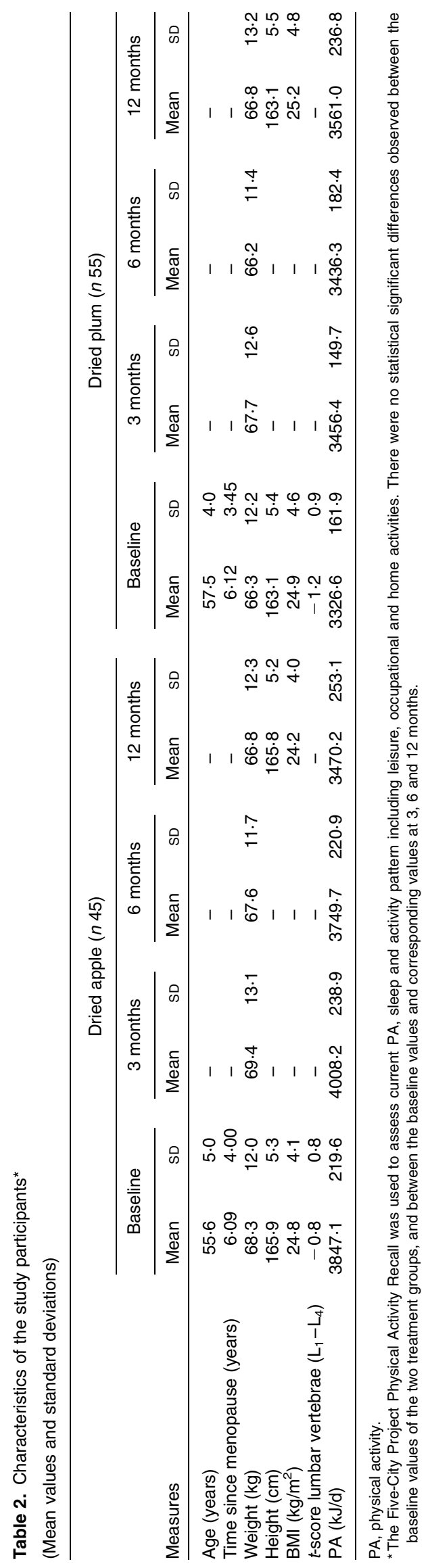


Table 3. Daily nutrient intake calculated from a $7 \mathrm{~d}$ FFQ of women at baseline, 3,6 and 12 months supplementation with $100 \mathrm{~g}$ dried plum or $75 \mathrm{~g}$ dried apple daily (Mean values and standard deviations)

\begin{tabular}{|c|c|c|c|c|c|c|c|c|c|c|c|c|c|c|c|c|}
\hline \multirow[b]{3}{*}{ Daily intake } & \multicolumn{8}{|c|}{ Dried apple $(n 45)$} & \multicolumn{8}{|c|}{ Dried plum ( $n 55)$} \\
\hline & \multicolumn{2}{|c|}{ Baseline } & \multicolumn{2}{|c|}{3 months } & \multicolumn{2}{|c|}{6 months } & \multicolumn{2}{|c|}{12 months } & \multicolumn{2}{|c|}{ Baseline } & \multicolumn{2}{|c|}{3 months } & \multicolumn{2}{|c|}{6 months } & \multicolumn{2}{|c|}{12 months } \\
\hline & Mean & SD & Mean & SD & Mean & SD & Mean & SD & Mean & SD & Mean & SD & Mean & SD & Mean & SD \\
\hline Total energy (kJ) & 7033 & 234 & 7314 & 305 & 7146 & 339 & 7557 & 267 & 6887 & 288 & 7498 & 255 & 7674 & 355 & 7733 & 322 \\
\hline \multicolumn{17}{|l|}{ Macronutrients } \\
\hline Protein $(\mathrm{g})$ & $72 \cdot 5$ & $3 \cdot 0$ & $66 \cdot 7$ & $2 \cdot 7$ & $67 \cdot 2$ & 3.5 & 73.6 & $2 \cdot 8$ & $67 \cdot 0$ & $2 \cdot 7$ & $72 \cdot 8$ & $2 \cdot 8$ & $68 \cdot 8$ & $2 \cdot 6$ & $72 \cdot 5$ & $3 \cdot 2$ \\
\hline Carbohydrate (g) & 212 & 8 & 236 & 10 & 227 & 9 & 231 & 9 & 205 & 10 & 233 & 9 & 256 & 17 & 233 & 10 \\
\hline Dietary fibre $(\mathrm{g})$ & $21 \cdot 2$ & $1 \cdot 2$ & 24.5 & $1 \cdot 1$ & $22 \cdot 7$ & $1 \cdot 2$ & $25 \cdot 0$ & 1.4 & $21 \cdot 2$ & $1 \cdot 1$ & $24 \cdot 4$ & 1.3 & $26 \cdot 6$ & $1 \cdot 8$ & $26 \cdot 2$ & 1.7 \\
\hline Total fat $(\mathrm{g})$ & $61 \cdot 1$ & $3 \cdot 2$ & 60.9 & 4.8 & 60.9 & 4.6 & $67 \cdot 1$ & 3.9 & $62 \cdot 3$ & $3 \cdot 1$ & 63.8 & 3.1 & 60.5 & $2 \cdot 7$ & $70 \cdot 7$ & 4.6 \\
\hline Saturated fat (g) & $18 \cdot 8$ & 1.0 & $20 \cdot 6$ & 2.5 & $19 \cdot 8$ & 1.7 & $21 \cdot 0$ & $1 \cdot 2$ & $19 \cdot 2$ & 1.3 & $19 \cdot 7$ & $1 \cdot 1$ & $19 \cdot 2$ & $1 \cdot 0$ & $21 \cdot 7$ & 1.4 \\
\hline Monounsaturated fat $(\mathrm{g})$ & $21 \cdot 4$ & 1.5 & $19 \cdot 4$ & 1.4 & $20 \cdot 6$ & 1.8 & $22 \cdot 8$ & 1.8 & $21 \cdot 8$ & 1.2 & $21 \cdot 3$ & $1 \cdot 2$ & 20.5 & $1 \cdot 1$ & $23 \cdot 4$ & 1.8 \\
\hline Polyunsaturated fat (g) & 11.6 & 0.9 & 11.0 & 0.8 & $11 \cdot 1$ & 0.9 & $12 \cdot 7$ & $1 \cdot 1$ & 11.6 & 0.6 & $11 \cdot 7$ & 0.7 & $11 \cdot 2$ & 0.7 & 13.9 & 1.1 \\
\hline Trans-fat (g) & 0.5 & $0 \cdot 1$ & 0.4 & $0 \cdot 1$ & 0.4 & $0 \cdot 1$ & 0.8 & $0 \cdot 1$ & 0.4 & $0 \cdot 1$ & $0 \cdot 8$ & 0.2 & 0.7 & 0.1 & 0.8 & 0.2 \\
\hline Total cholesterol $(\mathrm{mg})$ & $196 \cdot 5$ & 14.5 & 195.9 & $15 \cdot 8$ & $227 \cdot 2$ & $18 \cdot 0$ & 221.4 & $14 \cdot 0$ & 202.5 & $12 \cdot 5$ & 224.8 & $13 \cdot 3$ & 223.9 & $12 \cdot 3$ & 249.1 & $17 \cdot 4$ \\
\hline \multicolumn{17}{|l|}{ Vitamins } \\
\hline $\mathrm{A}(\mu \mathrm{g} \mathrm{RAE})$ & 3494 & 265 & 2793 & 290 & 2766 & 290 & 3406 & 410 & 3246 & 279 & 3492 & 300 & 3071 & 247 & 3916 & 427 \\
\hline$C(\mathrm{mg})$ & 107 & 8 & 93 & 8 & 82 & 8 & 93 & 7 & 93 & 8 & 95 & 11 & 98 & 12 & 99 & 68 \\
\hline$D(\mu \mathrm{g})$ & 2.57 & 0.27 & $2 \cdot 67$ & 0.27 & 2.52 & 0.25 & $2 \cdot 65$ & 0.30 & 2.42 & 0.30 & 2.50 & 0.27 & $2 \cdot 40$ & 0.25 & $2 \cdot 57$ & 0.25 \\
\hline$E(\mu \mathrm{g})$ & 3758 & 335.5 & 4496 & 335.5 & 4026 & 402 & 5033 & 536 & 5771 & 1208 & 4966 & 469 & 4832 & 402 & 5973 & 872 \\
\hline $\mathrm{K}(\mu \mathrm{g})$ & $115 \cdot 6$ & 11.6 & $168 \cdot 0$ & $16 \cdot 8$ & $135 \cdot 0$ & $27 \cdot 3$ & 174.5 & 30.5 & $209 \cdot 2$ & $21 \cdot 6$ & $170 \cdot 4$ & $17 \cdot 3$ & $178 \cdot 0$ & $16 \cdot 6$ & 198.4 & $21 \cdot 8$ \\
\hline \multicolumn{17}{|l|}{ Minerals (mg) } \\
\hline $\mathrm{Ca}$ & 808 & 47 & 896 & 52 & 806 & 57 & 861 & 54 & 927 & 142 & 779 & 62 & 762 & 42 & 885 & 63 \\
\hline $\mathrm{Fe}$ & 14.7 & $1 \cdot 2$ & 14.4 & 0.9 & 13.5 & 0.7 & 14.9 & 0.9 & $16 \cdot 8$ & $2 \cdot 5$ & $15 \cdot 0$ & $1 \cdot 0$ & $15 \cdot 0$ & 0.9 & $15 \cdot 7$ & 1.2 \\
\hline $\mathrm{Mg}$ & 245 & 10 & 288 & 12 & 263 & 16 & 301 & 17 & 296 & 13 & 285 & 14 & 310 & 19 & 321 & 21 \\
\hline$P$ & 997 & 42 & 1172 & 53 & 1048 & 63 & 1155 & 49 & 1120 & 50 & 1079 & 51 & 1087 & 44 & 1161 & 64 \\
\hline $\mathrm{K}$ & 2661 & 97 & 2810 & 115 & 2643 & 129 & 2916 & 121 & 2957 & 131 & 2687 & 144 & 3030 & 147 & 3025 & 149 \\
\hline $\mathrm{Zn}$ & $7 \cdot 1$ & 0.3 & $8 \cdot 2$ & 0.4 & 7.4 & 0.4 & 8.6 & 0.4 & $10 \cdot 1$ & $2 \cdot 0$ & 7.5 & 0.3 & $8 \cdot 2$ & 0.5 & $8 \cdot 1$ & 0.4 \\
\hline
\end{tabular}

RAE, retinol activity equivalents.

*Analyses do not include nutrients, $\mathrm{Ca}$ and vitamin $\mathrm{D}$ from the supplements used by the participants. There were no statistically significant differences observed between the baseline values of the two treatment groups, and between the baseline values and corresponding values at 3,6 and 12 months. 
Physical activity levels were assessed at baseline, 3, 6 and 12 months, and as expected, there were no significant differences in their activity levels throughout the study (Table 2).

\section{Bone mineral density}

Both dried fruit regimens had bone-protective effects as indicated by positive changes from baseline in ulna, spine, femoral neck, total hip and whole-body BMD (Fig. 2). Dried plum had more pronounced effects on BMD of the ulna and spine, as the increases were significantly different between the two treatments.

\section{Serum parameters of relevance to bone metabolism}

Dried plum consumption resulted in a time-dependent reduction in serum BALP levels of postmenopausal women at 3-, 6- and 12-month time intervals, and this reduction became significant at 12 months compared with baseline (Table 4). In the dried apple group, serum BALP levels increased significantly in the first 6 months of the study and decreased at 12 months. BALP levels were significantly lower in the dried plum group at 6 months compared with the dried apple group.

OC, a marker of bone turnover, decreased in a time-dependent manner in the dried plum group, while it increased in the dried apple group over time and this increase reached a significant level at 6 months (Table 4). Serum OC levels were significantly lower in the dried plum group compared with the dried apple group at 3- and 12-month time points.

TRAP5b, a specific marker of bone resorption, decreased significantly in the dried plum group at 3 months and stayed at the same level at the 6- and 12-month time points (Table 4). TRAP5b did not change signficantly in the dried apple group; however, it increased at the 12-month time point.

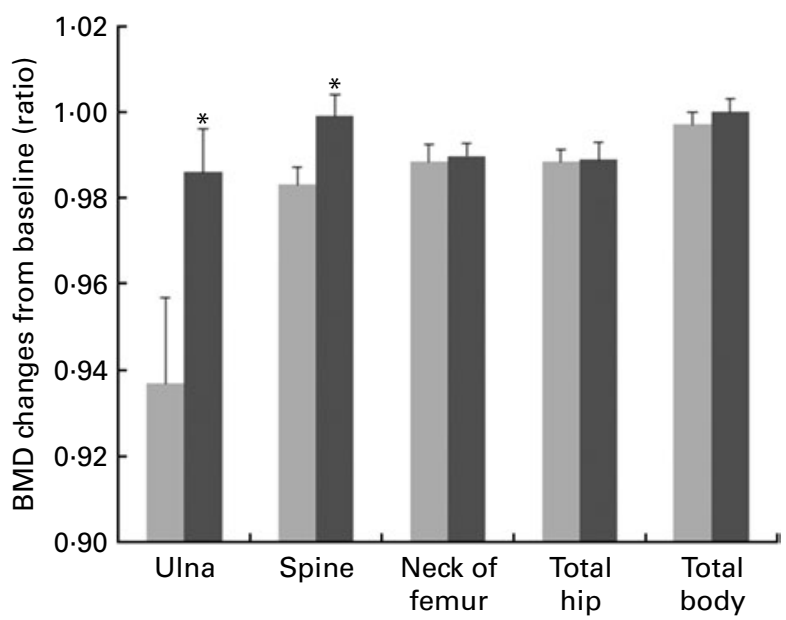

Fig. 2. Bone mineral density (BMD) changes from baseline in ulna, spine femoral neck, total hip and total body after a 1-year consumption of dried apple ( $\square$ ) or dried plum ( $\square$ ). Values are means, with standard deviations represented by vertical bars. * Mean values were significantly different between the treatment groups $(P<0.05)$.

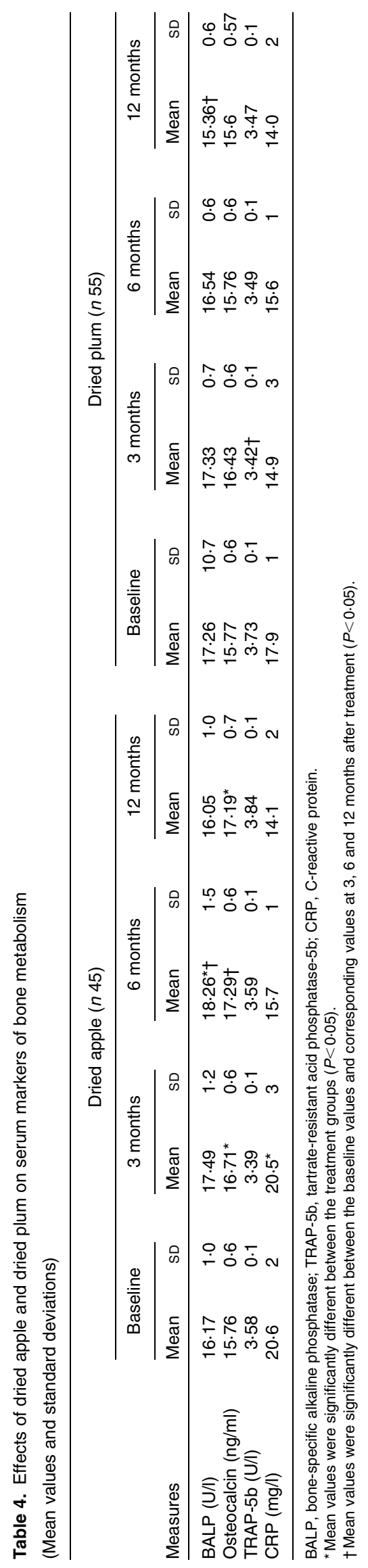


Serum CRP, a marker of inflammation, decreased in the dried plum treatment group after 3 months and remained the same thereafter. On the other hand, CRP levels were the same after 3 months in the dried apple group and decreased thereafter. Serum CRP levels were significantly lower in the dried plum group compared with the dried apple group at 3 months (Table 4).

\section{Discussion}

In a recent review ${ }^{(21)}$, we summarised the prior evidence of bone-protective effects of dried plum and speculated the mechanisms of action. Earlier findings strongly suggest that dried plum has a potent effect on bone in terms of both prevention and reversal as is evident by higher bone densities, mineral contents, percentage trabecular bone area and tendency to reduce marrow space in the long bone of rats $^{(9-13)}$. The findings of the present study confirmed the ability of dried plum in improving BMD in postmenopausal women. Postmenopausal women in this age range are prone to accelerated bone loss. Women potentially lose up to $50 \%$ of their trabecular bone and $30 \%$ of their cortical bone after reaching peak bone mass in which half of it occurs during the first 10 years from the onset of menopause ${ }^{(22)}$. To our knowledge, the present study is the first to clearly indicate that postmenopausal women benefit from consuming dried plum in terms of BMD.

As expected, both groups started with similar baseline characteristics including age, time since menopause, weight, height, BMI and lumbar $t$-score, indicative of complete randomisation. Although the women on both regimens consumed $1004 \mathrm{~kJ} / \mathrm{d}$ more energy (albeit not significantly different from baseline energy consumption in both regimens), neither their weight nor their BMI was significantly affected. This may be due to the high fibre contents of the dried fruits. Other investigators have reported lack of weight gain despite higher energy intakes associated with higher fibre content, such as flaxseed ${ }^{(23-25)}$. These findings are also in accordance with our earlier findings ${ }^{(17,24)}$, in which women did not gain weight by consuming similar regimens for 3 months.

The present data indicate that postmenopausal women on both dried plum and dried apple regimens did not lose bone. However, the gain in BMD for ulna and spine was significantly higher in the dried plum group than in the dried apple group. These findings suggest that dried plum is particularly effective in reducing the risk of fracture in ulna and spine as indicated by higher BMD in these sites. These results support the findings that, in general, consumption of fruits and vegetables is beneficial to bone health ${ }^{(3,5-7)}$. However, the majority of studies that have linked fruit and vegetable consumption to better bone health speculated that the beneficial effects of fruits and vegetables on bone were due to a shift in the acid-base balance ${ }^{(6)}$. Nonetheless, more recent studies have suggested that the beneficial effects of fruit and vegetable consumption on bone may be due to their contents such as vitamin $\mathrm{C}$, vitamin $\mathrm{K}, \mathrm{K}$ and phytochemicals. The present study does not address the osteoprotective mechanism of dried plums. Dried plums are rich in phenolic compounds such as neochlorogenic acid and chlorogenic acid, which act as antioxidants ${ }^{(26,27)}$. Antioxidants that scavenge potentially damaging free radicals have been shown to prevent bone loss ${ }^{(28,29)}$. Dried plums also contain higher amounts of boron than most fruits. Boron has been shown to modulate bone and $\mathrm{Ca}$ metabolism ${ }^{(30)}$, and play an important role in preserving $\mathrm{BMD}^{(31)}$

In addition, dried plums contain high amounts of vitamin $\mathrm{K}^{(32)}$. Vitamin $\mathrm{K}$ influences bone health by improving Ca balance $^{(33)}$ and is also a cofactor needed for $\gamma$-carboxylation of OC. $\gamma$-Carboxylated OC promotes normal bone mineralisation by regulating the growth of hydroxyapatite crystals ${ }^{(34)}$. However, it should be emphasised that the bone-protective effects of fruits and vegetables including dried plum should not be solely attributed to any one of their components, and consumption of whole fruits and vegetables should be promoted.

From a mechanistic point of view, we measured both markers of bone formation and bone resorption. In earlier studies, BALP and OC were considered to be markers of bone formation; however, more recent studies ${ }^{(34-37)}$ have suggested that both are considered as markers of bone turnover rather than bone formation. Therefore, our overall findings suggest that dried plum improves bone mass by slowing down the rate of bone turnover. Nonetheless, it should be noted that in order for dried plum to have bone reversal effects, it must suppress the rate of bone resorption more so than the rate of bone formation. Although this is speculative, in order to examine this notion, one must use bone biopsies to assess static and dynamic histomorphometry. Particularly, quantitative histomorphometry is necessary to assess the effects of dried plum on the appearance of the cellular components, the presence or absence of woven bone and marrow fibrosis. Another explanation for the bone reversal property of dried plum may be through the suppression of chronic inflammation, as dried plum was able to significantly lower serum CRP level after 3 months and reached a plateau thereafter. CRP is known to be linked to a number of chronic diseases including osteoporosis ${ }^{(37-40)}$. Therefore, the efficacy of dried plum in lowering serum CRP levels may have far greater implications than just influencing bone turnover. The present study suggests that the longer-term consumption of dried plums increases BMD in postmenopausal women, in part, due to suppressing the rate of bone turnover and perhaps through lowering CRP levels.

\section{Acknowledgements}

The present study was supported by the National Research Initiative of the USDA Cooperative State Research, Education and Extension Service (grant no. 2005-35200-17053). The authors thank the California Dried Plum Board (Sacramento, CA, USA) for providing us with dried plums, and we gratefully acknowledge Bayer HealthCare, LLC (Morristown, NJ, USA), for supplying $\mathrm{Ca}$ and vitamin $\mathrm{D}$ supplements for the study. All authors contributed to the data analysis and interpretation, drafting the manuscript and approving the final version submitted. There are no conflicts of interest. 


\section{References}

1. National Osteoporosis Foundation (2010) National Osteoporosis Foundation. www.nof.org

2. Barnes PM, Powell-Griner E, McFann K, et al. (2004) Complementary and alternative medicine use among adults: United States, 2002. Adv Data 343, 1-19.

3. Macdonald HM, New SA, Golden MH, et al. (2004) Nutritional associations with bone loss during the menopausal transition: evidence of a beneficial effect of calcium, alcohol, and fruit and vegetable nutrients and of a detrimental effect of fatty acids. Am J Clin Nutr 79, 155-165.

4. Prynne CJ, Mishra GD, O'Connell MA, et al. (2006) Fruit and vegetable intakes and bone mineral status: a cross sectional study in 5 age and sex cohorts. Am J Clin Nutr 83, 1420-1428.

5. Tucker KL, Hannan MT, Chen H, et al. (1999) Potassium, magnesium, and fruit and vegetable intakes are associated with greater bone mineral density in elderly men and women. Am J Clin Nutr 69, 727-736.

6. Tucker KL, Hannan MT \& Kiel DP (2001) The acid-base hypothesis: diet and bone in the Framingham Osteoporosis Study. Eur J Nutr 40, 231-237.

7. Tucker KL, Chen H, Hannan MT, et al. (2002) Bone mineral density and dietary patterns in older adults: the Framingham Osteoporosis Study. Am J Clin Nutr 76, 245-252.

8. Muhlbauer RC, Lozano A, Reinli A, et al. (2003) Various selected vegetables, fruits, mushrooms and red wine residue inhibit bone resorption in rats. J Nutr 133, 3592-3597.

9. Franklin M, Bu SY, Lerner MR, et al. (2006) Dried plum prevents bone loss in a male osteoporosis model via IGF-I and the RANK pathway. Bone 39, 1331-1342.

10. Deyhim F, Stoecker BJ, Brusewitz GH, et al. (2005) Dried plum reverses bone loss in an osteopenic rat model of osteoporosis. Menopause 12, 755-762.

11. Smith BJ, Ethriedge A, Lucas EA, et al. (2003) Dried plum promotes bone recovery comparable to parathyroid hormone in osteopenic rats following hind limb unloading. J Bone Min Res 18, S277.

12. Bu SY, Lucas EA, Franklin M, et al. (2007) Comparison of dried plum supplementation and intermittent PTH in restoring bone in osteopenic orchidectomized rats. Osteoporos Int 18, 931-942.

13. Arjmandi BH, Lucas EA, Juma S, et al. (2001) Dried plum prevent ovariectomy-induced bone loss in rats. JANA 4, 50-56.

14. Arjmandi BH, Birnbaum R, Goyal NV, et al. (1998) Bone-sparing effect of soy protein in ovarian hormone-deficient rats is related to its isoflavone content. Am J Clin Nutr 68, 1364S-1368S.

15. Arjmandi BH (2001) The role of phytoestrogens in the prevention and treatment of osteoporosis in ovarian hormone deficiency. I Am Coll Nutr 20, 398S-402S.

16. Lucas EA, Wild RD, Hammond LJ, et al. (2002) Flaxseed improves lipid profile without altering biomarkers of bone metabolism in postmenopausal women. J Clin Endocrinol Metab 87, 1527-1532.

17. Arjmandi BH, Khalil DA, Lucas EA, et al. (2002) Dried plums improve indices of bone formation in postmenopausal women. $J$ Womens Health Gend Based Med 11, 61-68.

18. Lane NE, Haupt D, Kimmel DB, et al. (1999) Early estrogen replacement therapy reverses the rapid loss of trabecular bone volume and prevents further deterioration of connectivity in the rat. J Bone Miner Res 14, 206-214.

19. Kalu DN, Arimandi BH, Liu CC, et al. (1994) Effects of ovariectomy and estrogen on the serum levels of insulin-like growth factor-I and insulin-like growth factor binding protein-3. Bone Miner 25, 135-148.

20. Sallis JF, Fortmann SP, Solomon DS, et al. (1984) Increasing returns of physician surveys. Am J Public Health 74, 1043.
21. Hooshmand S \& Arjmandi BH (2009) Viewpoint: dried plum, an emerging functional food that may effectively improve bone health. Ageing Res Rev 8, 122-127.

22. Finkelstein JS, Brockwell SE, Mehta V, et al. (2008) Bone mineral density changes during the menopause transition in a multiethnic cohort of women. $J$ Clin Endocrinol Metab 93, 861-868.

23. Gustafsson IB, Vessby B, Ohrvall M, et al. (1994) A diet rich in monounsaturated rapeseed oil reduces the lipoprotein cholesterol concentration and increases the relative content of $n$-3 fatty acids in serum in hyperlipidemic subjects. $A m J$ Clin Nutr 59, 667-674.

24. Patade A, Devareddy L, Lucas EA, et al. (2008) Flaxseed reduces total and LDL cholesterol concentrations in Native American postmenopausal women. I Womens Health (Larchmt) 17, 355-366.

25. Vahouny GV, Satchithanandam S, Chen I, et al. (1988) Dietary fiber and intestinal adaptation: effects on lipid absorption and lymphatic transport in the rat. Am J Clin Nutr 47, 201-206.

26. Kayano S, Kikuzaki H, Fukutsuka N, et al. (2002) Antioxidant activity of prune (Prunus domestica L.) constituents and a new synergist. J Agric Food Chem 50, 3708-3712.

27. Nakatani N, Kayano S, Kikuzaki H, et al. (2000) Identification, quantitative determination, and antioxidative activities of chlorogenic acid isomers in prune (Prunus domestica L.). J Agric Food Chem 48, 5512-5516.

28. Basu S, Michaelsson K, Olofsson H, et al. (2001) Association between oxidative stress and bone mineral density. Biochem Biophys Res Commun 288, 275-279.

29. Garrett IR, Boyce BF, Oreffo RO, et al. (1990) Oxygen-derived free radicals stimulate osteoclastic bone resorption in rodent bone in vitro and in vivo. J Clin Invest 85, 632-639.

30. Nielsen FH, Hunt CD, Mullen LM, et al. (1987) Effect of dietary boron on mineral, estrogen, and testosterone metabolism in postmenopausal women. FASEB J 1, 394-397.

31. Nielsen FH (1994) Biochemical and physiologic consequences of boron deprivation in humans. Environ Health Perspect 102, Suppl. 7, 59-63.

32. Dismore ML, Haytowitz DB, Gebhardt SE, et al. (2003) Vitamin $\mathrm{K}$ content of nuts and fruits in the US diet. $J$ Am Diet Assoc 103, 1650-1652.

33. Iwamoto J, Takeda $\mathrm{T}$ \& Sato $\mathrm{Y}$ (2004) Effects of vitamin $\mathrm{K}_{2}$ on osteoporosis. Curr Pharm Des 10, 2557-2576.

34. Azinge EC \& Bolarin DM (2006) Osteocalcin and bonespecific alkaline phosphatase in sickle cell haemoglobinopathies. Niger J Physiol Sci 21, 21-25.

35. Gundberg CM, Looker AC, Nieman SD, et al. (2002) Patterns of osteocalcin and bone specific alkaline phosphatase by age, gender, and race or ethnicity. Bone 31, 703-708.

36. Lenora J, Norrgren K, Thorsson O, et al. (2009) Bone turnover markers are correlated with total skeletal uptake of $99 \mathrm{mTc}-$ methylene diphosphonate (99mTc-MDP). BMC Med Phys 9, 3.

37. Wang J, Pei F, Tu C, et al. (2007) Serum bone turnover markers in patients with primary bone tumors. Oncology 72, 338-342.

38. Ding C, Parameswaran V, Udayan R, et al. (2008) Circulating levels of inflammatory markers predict change in bone mineral density and resorption in older adults: a longitudinal study. J Clin Endocrinol Metab 93, 1952-1958.

39. Lund HL, Nafstad P, Olsen I, et al. (2009) C-reactive protein variations for different chronic somatic disorders. Scand $J$ Public Health 37, 640-646.

40. Maugeri D, Russo MS, Franze C, et al. (1998) Correlations between C-reactive protein, interleukin-6, tumor necrosis factor-alpha and body mass index during senile osteoporosis. Arch Gerontol Geriatr 27, 159-163. 